\title{
ESTIMACIÓN DEL ÍNDICE DE ÁREA FOLIAR EN PLANTACIONES DE Eucalyptus grandis W. Hill.
}

\section{Leaf area index estimation of Eucalyptus grandis W. Hill. in plantations}

Palabras clave: modelo matemático, Plant Canopy Analyzer, plantaciones.

Key words: mathematical model, Plant Canopy Analyzer, plantation.

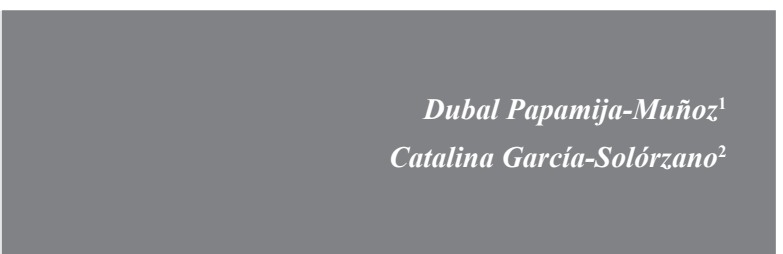

\section{RESUMEN}

Se realizó la estimación del índice de área foliar en plantaciones de Eucalyptus grandis W.Hill. en cuatro fincas de la empresa Smurfit Kappa Cartón de Colombia (SKCC), tres de ellas ubicadas en el municipio de Popayán, Cauca, y una ubicada en el municipio de Restrepo, Valle del Cauca. Cada finca presentaba tres parcelas fertilizadas y tres parcelas no fertilizadas, con 64 individuos en cada parcela. Para dicha estimación se utilizaron tres métodos: Plant Canopy Analyzer 2000 (PCA 2000), fotografía plana a través del software PIPEcv y un método destructivo, con el cual se generó un modelo matemático. Para los dos primeros métodos se realizaron mediciones bimensuales durante un año y para el tercero se realizó una clasificación diamétrica para cortar los árboles en la última medición. La estimación del índice de área foliar para las tres metodologías fue en promedio 2.01 para el PCA 2000, 3.12 para el software PIPEcv y 2.83 con el modelo matemático, lo cual corresponde a los promedios y rangos de índice de área foliar, obtenidos con cada metodología en el total de fincas. Los análisis permitieron establecer que las tres metodologías desarrolladas en la investigación no tuvieron una estrecha relación.

\begin{abstract}
We estimated leaf area index (LAI) in Eucalyptus grandis W.Hill. plantations in four farms in the Smurfit Kappa Carton de Colombia (SKCC) with three farms located in the city of Popayan (Cauca) and one located in the municipality of Restrepo (Valle del Cauca). Each farm had three fertilized and three unfertilized plots with 64 individuals in each. We used three methods, Plant Canopy Analyzer 2000 (PCA 2000), flat photograph PIPEcv software and a destructive method, which was generated using a mathematical model. The first two methods were measured bimonthly for a year and the final method required trees being cut to measure their diameter. Estimation of leaf area index -was 2.01 for PCA 2000, 3.12 for PIPEcv and 2.83 for the mathematical model. These values correspond to the average and range of leaf area indices obtained for each method on all farms. Statistically the three methodologies developed in this study were not closely related.
\end{abstract}

\section{INTRODUCCIÓN}

Los diferentes ecosistemas boscosos nativos y cultivados presentan una estructura foliar denomi-

Grupo de investigación para el desarrollo rural TULL, Universidad del Cauca. Popayán, Colombia. dubalpapamija@gmail.com

Grupo de investigación para el desarrollo rural TULL, Facultad de Ciencias Agropecuarias "Las Guacas", Universidad del Cauca. Calle 5 No.

4-70, Popayán, Colombia. catalinagarcia@unicauca.edu.co. Autor para correspondencia. 
nada dosel, la cual está conformada básicamente por el conjunto de las copas de los árboles que lo componen (Cabrelli et al., 2006). Dicha estructura varía dependiendo del tipo de bosque tanto en el plano horizontal, como vertical, y de características como la composición florística, densidad, distribución espacial de los árboles, tipo de copa, estado fenológico de las especies, edad y manejo (Lieberman et al., 1989; Pukkala et al., 1991). El dosel forestal está directamente relacionado con la atenuación de la cantidad de radiación fotosintéticamente activa (Rafa) que llega al piso del bosque (Pukkala et al., 1991), de ahí que el índice de área foliar (LAI) sea un indicador de la producción de un bosque. También es ampliamente reconocido que bajo el dosel de un bosque se genera un microclima que se halla fuertemente regulado por la cantidad y calidad de radiación solar que ingresa a este (Hutchison \& Matt, 1977; Chazdon \& Fetcher, 1984; Kellomaki et al., 1985; Poulson \& Platt, 1989; Canham et al., 1990), el cual presenta a nivel del suelo una alta variabilidad espacial y temporal (Pukkala et al., 1991; Hardy et al., 2004).

En el caso de las plantaciones forestales de Eucalyptus grandis W.Hill., la estructura foliar se encuentra de una forma más ordenada y homogénea, debido al cuidado y manejo silvicultural dado durante todo el periodo de siembra, hasta el momento de la cosecha. En las plantaciones de $E$. grandis, el LAI varía principalmente por variables como la disponibilidad de nutrientes en el suelo, condiciones y calidad de luz, estado fitosanitario y de desarrollo del árbol. Es importante estimar este índice, pues, al funcionar como un indicador de la producción de una plantación, permite establecer la existencia de diferencias en la producción de acuerdo a la fertilización recibida.

Esta investigación tuvo como objetivo estimar el LAI en plantaciones bajo diferentes tratamientos silviculturales (fertilizaciones), mediante los métodos indirectos Plant Canopy Analyzer (PCA 2000) y fotografía plana. Asimismo, se pretendió encontrar un factor de conversión para ajustar la metodología de fotografía plana al PCA 2000 y finalmente realizar una prueba destructiva con el fin de validar la información recolectada en campo con los dos métodos mencionados para formular un modelo matemático que permita estimar el LAI, a través de variables de fácil medición.

\section{MATERIALES Y MÉTODOS}

\section{ÁREA DE ESTUDIO}

La investigación se desarrolló en cuatro fincas pertenecientes a la empresa Smurfit Kappa Cartón de Colombia, la cual, en convenio con la Universidad del Cauca, permitió la investigación en los departamentos del Valle del Cauca y el Cauca. Las mediciones se realizaron en parcelas en donde se tenían establecidos ensayos de fertilización con los respectivos controles; es decir, aquellas que no recibieron ningún tratamiento químico o de fertilización. La caracterización general de los lugares de estudio se presenta a continuación:

\section{Finca Chupillauta}

Está ubicada en las afueras del sector urbano de Popayán (2॰31'01" latitud norte, 76³7'17' longitud oeste), correspondiente al subnúcleo forestal Chupillauta, así denominado por la empresa SKCC. Se encuentra a $1736.3 \mathrm{~m}$ de altitud y tiene una precipitación promedio anual de $2177 \mathrm{~mm}$. La finca está situada sobre la unidad de suelos Pubenza.

\section{Finca El Pital}

Está ubicada en el municipio de Cajibío, Cauca, (2॰35'43” latitud norte, $76^{\circ} 35^{\prime} 15^{\prime}$ 'longitud oeste), pertenece al subnúcleo forestal San José, se encuentra a $1793 \mathrm{~m}$ de altitud y presenta una precipitación promedio anual de $2244 \mathrm{~mm}$. Esta finca también está dentro de la unidad de suelos Pubenza.

\section{Finca Don Miguel}

Está ubicada en el municipio de Sotará ( $2^{\circ} 17^{\prime} 27^{\prime \prime}$ latitud norte, 76³9'46" longitud oeste), perteneciente al subnúcleo forestal Juanambú dentro del núcleo Salinas, así denominado por 
la empresa. Se encuentra a $2069 \mathrm{~m}$ de altitud y presenta una precipitación promedio anual de $2539 \mathrm{~mm}$. Esta finca, pertenece a la unidad de suelos Salinas.

\section{Finca Rancho Grande}

Está ubicada en el municipio de Restrepo Valle (351'43" latitud norte, $76^{\circ} 30^{\prime} 48^{\prime}$ ' longitud oeste), esta finca se atañe al núcleo de la zona centro de la empresa SKCC. Tiene una elevación de 1455 y una precipitación media anual de $1167 \mathrm{~mm}$, pertenece a la unidad de suelos Restrepo y al orden taxonómico de los inceptisoles.

\section{DISEÑO EXPERIMENTAL Y MEDICIONES}

Las parcelas de medición en cada una de las fincas se establecieron en bloques completamente escogidos al azar generalizado, donde los tratamientos se repitieron dentro de cada bloque. La fertilización consistió en la aplicación bianual de $180 \mathrm{~kg} \mathrm{ha}^{-1}$ de nitrógeno, $18 \mathrm{~kg} \mathrm{ha}^{-1}$ de fósforo $\mathrm{y}$ $1.8 \mathrm{~kg} \mathrm{ha}^{-1}$ de boro. La aplicación se realizó en todas las fincas desde su establecimiento hasta el momento de la medición, la cual se realizó a los 7 años de edad. Para efectos de la investigación cada finca correspondió a un bloque.

Las mediciones del LAI se llevaron a cabo cada dos meses, para ello se usó el PCA 2000 (LI-COR, 1992) con un viewcap de $30^{\circ}$ en seis puntos por parcela con 5 réplicas por punto (Figura 1), y para la validación de LAI se tomaron nueve fotografías por parcela con un método operativo, utilizando una cámara digital de forma perpendicular al suelo, con la lente apuntando hacia las copas de los árboles (Figura 2), a una altura de $1.30 \mathrm{~m}$.

Las mediciones hechas en cada finca con el PCA 2000 fueron tomadas en horas de la mañana (para minimizar el efecto de la luz, ya que el PCA 2000 está diseñado para estimar el LAI en condiciones de luz difusa) tanto en las parcelas fertilizadas, como en las testigo. Para cada parcela se dejó una zona buffer o de amortiguamiento correspondiente a tres líneas de árboles a lado y lado de la parcela, con el fin de garantizar que el terreno medido era realmente la parcela objeto de estudio (Figura 1). El PCA 2000 se programó con una secuencia de puntos A (above) y puntos B (below), se tomaron mediciones a cielo abierto o fuera del dosel (denominados como puntos A) y otras bajo el dosel (denominados como puntos B). El procedimiento para cada parcela fue tomar tres puntos A y 30 puntos $\mathrm{B}$, los cuales se repartieron en 6 puntos en la parcela.

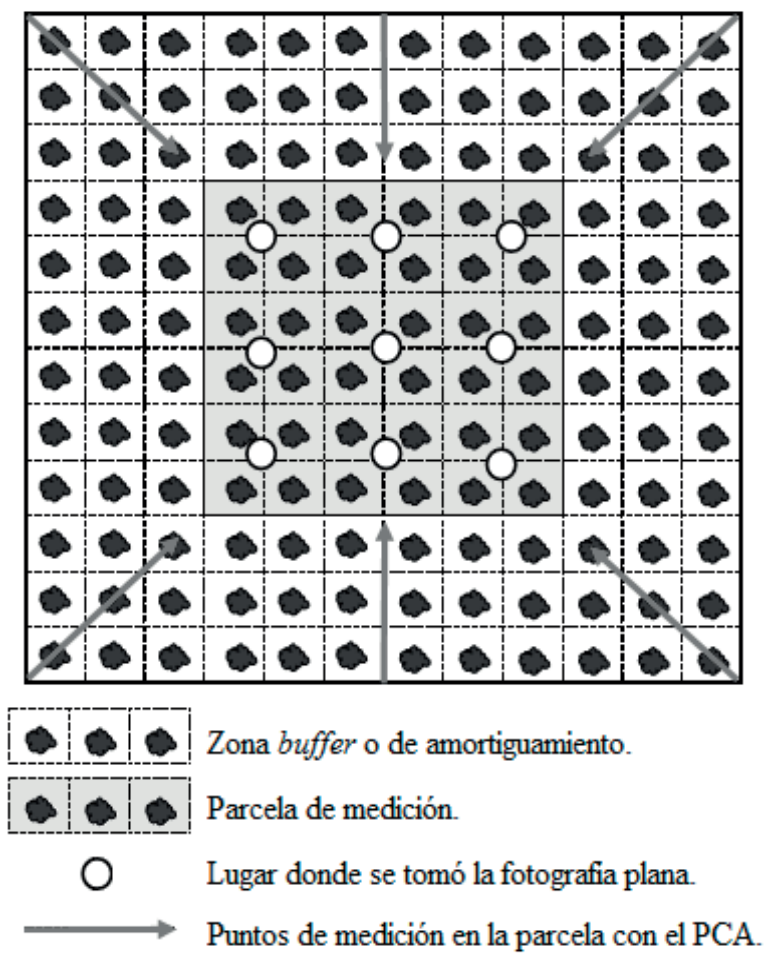

Figura 1. Modelo de la parcela donde se realizaron las mediciones con el Plant Canopy Analyzer y toma de las fotografías, para realizar la estimación del índice de área foliar en Eucalyptus grandis W.Hill.

Con las fotografías tomadas con la cámara digital se realizó una base de datos generada con el PIPEcv, el cual es un programa que procesa las imágenes y arroja un dato de índice de área foliar por cada foto (Figura 3). Una vez procesadas todas las fotos de una determinada parcela se promediaron los resultados para tener un estimativo del índice de área foliar, por parcela objeto de estudio. 


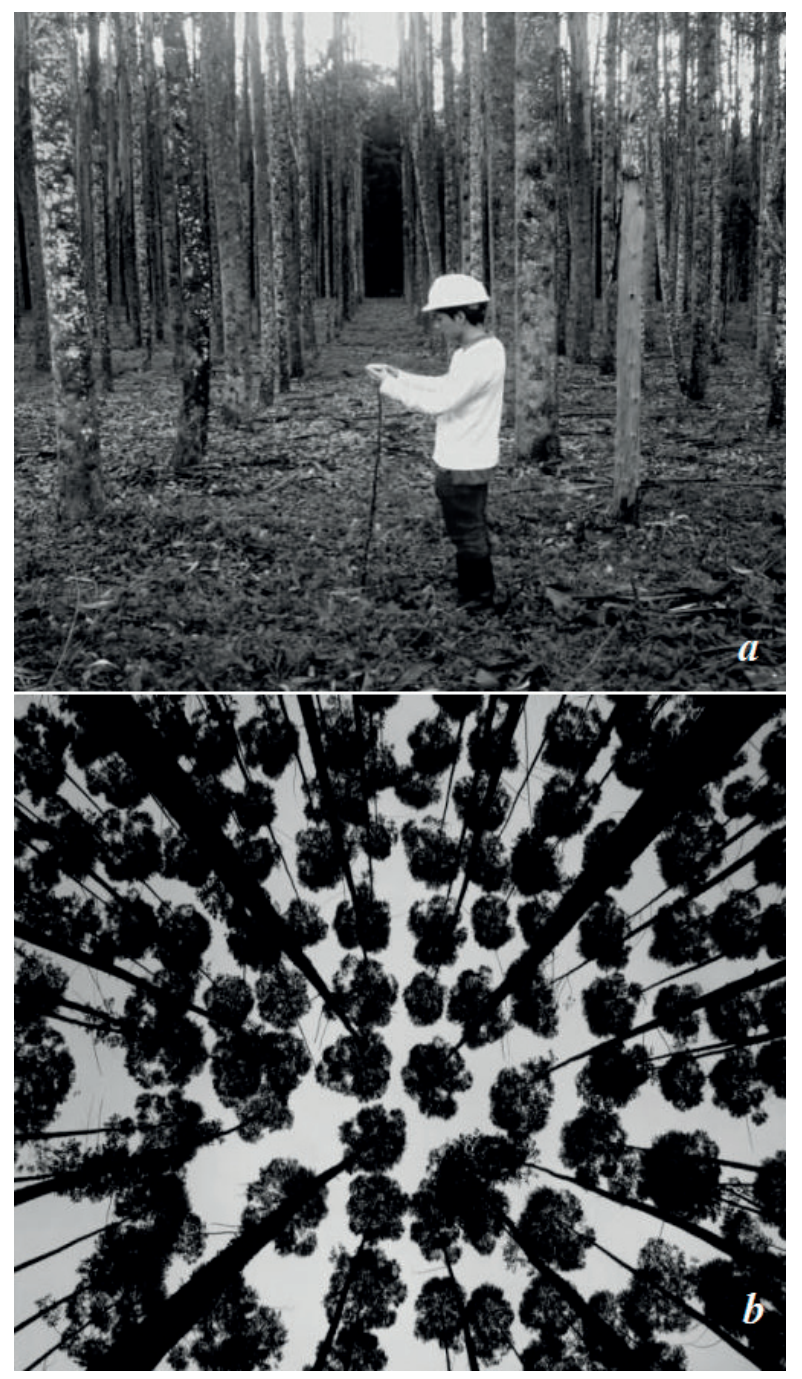

Figura 2. Toma de las fotografías digitales en la plantación de Eucalyptus grandis W. Hill en las parcelas fertilizadas y no fertilizadas en las fincas de la empresa Smurfit Kappa Cartón de Colombia. $\boldsymbol{a}$. Forma de tomar las fotografías con la cámara digital, $\boldsymbol{b}$. dosel de los árboles de Eucalyptus grandis W.Hill.

\section{MODELO MATEMÁTICO}

Para obtener el modelo matemático se cubicaron algunos árboles seleccionados por categorías diamétricas, se colectaron todas las hojas de cada uno de ellos y se pesaron en campo para obtener el peso fresco. Luego se tomó una muestra aleatoria de 30 hojas, las cuales fueron pesadas, marcadas y empacadas para ser llevadas a laboratorio y procesarlas en un escáner que midió directamente el área para cada una de las hojas.
Para encontrar elárea foliar de cada árbol se procedió a tomar el porcentaje de contenido de humedad de las 30 hojas, este porcentaje de humedad se usó con el fin de hallar su propio peso seco. Posteriormente, se realizó el mismo procedimiento para hallar el peso seco del total de hojas de cada árbol en kilogramos. A continuación se procedió a calcular el área foliar efectiva, para lo cual se tomó el área de las 30 hojas en metros cuadrados y se dividió entre su peso seco en kilogramos; de igual forma, se procedió para encontrar el área efectiva del total de hojas del árbol. Finalmente, para hallar el área foliar de cada árbol se multiplicó el área foliar efectiva total del árbol por el peso seco total de este, de esta manera quedó en unidades de área.
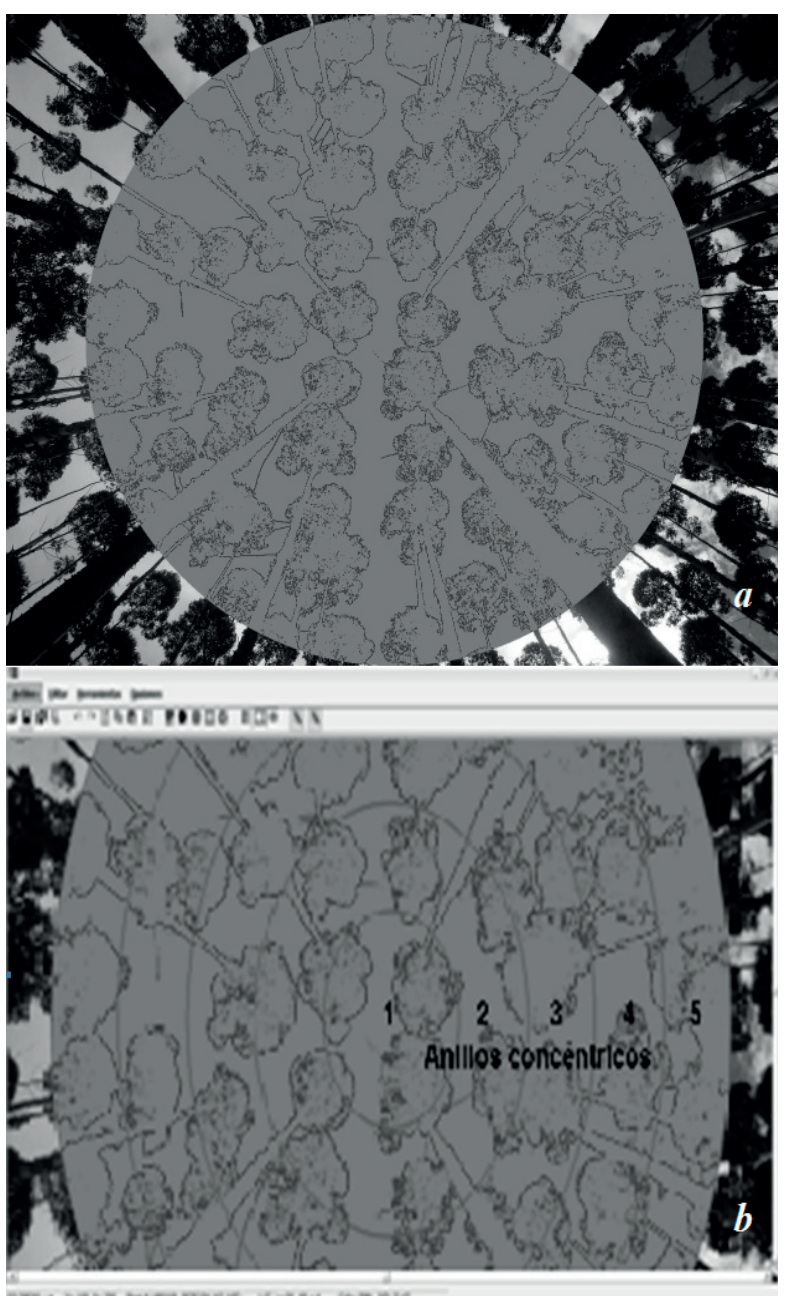

Figura 3. a. Fotografía plana del dosel de Eucalyptus grandis W.Hill. y procesada en el software PIPEcv, $\boldsymbol{b}$. división en los 5 anillos concéntricos para la estimación del LAI 
Cuando se encontró el área foliar de cada árbol se tomó su respectivo diámetro, medido a 130 $\mathrm{cm}$ de altura, y con estos datos se relacionaron las dos variables: dependiente (área foliar) y la independiente (diámetro). Los datos se llevaron al software CurveExpert Professional, con el fin de encontrar el modelo matemático de mejor ajuste, teniendo en cuenta tanto el coeficiente de correlación, como también el criterio biológico, para entender la dinámica de crecimiento de los árboles y la observación de la masa de puntos en el gráfico de dispersión.

Después de definir el modelo matemático, con el uso de Microsoft Excel ${ }^{\circledR}$ se organizaron los datos para llevarlos a la ecuación matemática con el fin de encontrar el área foliar de todos los árboles de cada parcela. Finalmente, para estimar el LAI a través del modelo, se realizó una sumatoria del área foliar de todos los árboles pertenecientes a la parcela y se dividió entre el área de suelo ocupada. Este mismo procedimiento se llevó a cabo con todas las parcelas y para cada una de las fincas.

\section{ANÁLISIS ESTADÍSTICO}

Con todos los datos obtenidos, tanto en campo, como en oficina, se realizaron gráficos de dispersión entre los métodos utilizados y un análisis de correlación lineal que permitió determinar si existía, o no, relación entre las variables de respuesta. Posteriormente se realizó un análisis de regresión lineal en el software SPSS ${ }^{\circledR}$ (versión 11.5 para Windows), diferenciando parcelas fertilizadas de las no fertilizadas y comparando el modelo matemático para la estimación del LAI con los otros dos métodos usados; es decir, con el PIPEcv y el PCA 2000.

Una vez realizada la estimación del LAI con el modelo matemático, se realizó una comparación de este método con el PCA 2000 y PIPEcv. Para ello se realizaron gráficos de dispersión con el software CurveExpert y regresiones lineales en el software SPSS (versión 11.5 para Windows). Lo anterior, con el fin de tabular los resultados de los parámetros estimados con sus errores estándar, en un intervalo de confianza del $95 \%$, para el intercepto y la pendiente, el límite superior e inferior para el total de los datos.

Después de verificar la normalidad de los datos y el cumplimiento de los supuestos estadísticos se realizó un análisis de varianza Anova con el software SPSS (versión 11.5 para Windows), con las diferencias encontradas entre los datos estimados con el modelo matemático frente a los estimados con el PCA 2000 y PIPEcv. Lo anterior se hizo con el fin de verificar si el modelo matemático guardaba relación alguna con las metodologías instrumentales.

\section{RESULTADOS}

Los valores estimados del índice de área foliar mediante las metodologías del PCA 2000, PIPEcv, y modelo matemático, que se presentan a continuación, son valores promediados de las mediciones realizadas para cada una de las fincas.

No se encontraron diferencias significativas para la estimación del LAI entre los tratamientos de fertilización con las tres metodologías aplicadas. Con el PIPEcv se reporta el mayor valor de LAI en promedio, para los dos tratamientos en todos los sitios estudiados. De esta forma, se encontró que la finca Don Miguel reporta el valor más alto de LAI, 3.64 para el tratamiento fertilizado y 3.50 para el tratamiento no fertilizado. Los valores de LAI más bajos reportados en los sitios de estudio se hallaron con el PCA 2000, particularmente en la finca Chupillauta; en ella se encontraron valores en promedio de LAI para el tratamiento fertilizado de 1.56 y para el tratamiento no fertilizado de 1.75. De igual forma, se puede observar que la metodología con menor variabilidad es la del modelo matemático y la de mayor variabilidad es la del PCA 2000 (Tabla 1).

\section{ESTIMACIÓN DEL ÍNDICE DE AREAFOLIAR CON EL PLANT CANOPY ANALYZER, EL PIPECV Y EL MODELO MATEMÁTICO}

El índice de área foliar de las fincas estudiadas estuvo en promedio, para la metodología del PCA 2000 , en 2.01; presentò valores máximos de 2.21 
Tabla 1. Estadísticos descriptivos de los tratamientos en las cuatro fincas estudiadas. F: Fertilizada, NF: No fertilizada, M: Promedio y $\sigma$ : Desviacion estandar

\begin{tabular}{|c|c|c|c|c|c|c|c|c|c|c|c|c|c|c|c|c|}
\hline \multirow{3}{*}{ Ítem } & \multicolumn{4}{|c|}{ Finca Chupillauta } & \multicolumn{4}{|c|}{ Finca Don Miguel } & \multicolumn{4}{|c|}{ Finca Don Miguel } & \multicolumn{4}{|c|}{$\begin{array}{c}\text { Finca Rancho } \\
\text { Grande }\end{array}$} \\
\hline & \multicolumn{2}{|c|}{$\mathrm{F}$} & \multicolumn{2}{|c|}{ NF } & \multicolumn{2}{|c|}{$\mathbf{F}$} & \multicolumn{2}{|c|}{ NF } & \multicolumn{2}{|c|}{$\mathbf{F}$} & \multicolumn{2}{|c|}{ NF } & \multicolumn{2}{|c|}{$\mathbf{F}$} & \multicolumn{2}{|c|}{ NF } \\
\hline & $\mathrm{M}$ & $\sigma$ & $M$ & $\sigma$ & $M$ & $\sigma$ & $M$ & $\sigma$ & $M$ & $\sigma$ & $M$ & $\sigma$ & $\mathrm{M}$ & $\sigma$ & $\mathrm{M}$ & $\sigma$ \\
\hline $\begin{array}{l}\text { LAI PCA } \\
2000\end{array}$ & 1.56 & 0.35 & 1.75 & 0.65 & 2.21 & 0.60 & 2.18 & 0.65 & 2.07 & 0.34 & 1.94 & 0.38 & 2.15 & 0.58 & 2.09 & 0.53 \\
\hline LAI PIPEcv & 2.61 & 0.61 & 2.42 & 0.57 & 3.64 & 0.65 & 3.50 & 0.70 & 2.98 & 0.55 & 3.10 & 0.52 & 3.34 & 0.42 & 3.22 & 0.36 \\
\hline $\begin{array}{l}\text { LAI Modelo } \\
\text { Matemático }\end{array}$ & 2.91 & 0.16 & 2.30 & 0.49 & 3.03 & 0.22 & 2.58 & 0.10 & 2.75 & 0.75 & 2.79 & 0.50 & 3.20 & 0.44 & 3.15 & 0.33 \\
\hline $\mathbf{N}$ válido & 1 & 4 & 2 & & 2 & 1 & 2 & 1 & 2 & & 2 & 1 & 2 & & 2 & 1 \\
\hline
\end{tabular}

para la finca Don Miguel en las parcelas fertilizadas y un valor mínimo para la finca Chupillauta con un valor de 1.56 en el tratamiento fertilizado. Con el PIPEcr se obtuvo un LAI promedio de 3.12, asimismo tuvo un valor máximo de 3.64 para el tratamiento fertilizado en la finca Don Miguel y un valor mínimo de 2.42 para la finca Chupillauta en la parcela no fertilizada. Con el modelo matemático se estimó un índice de área foliar promedio de 2.83; con un valor máximo de 3.20 para la parcela fertilizada de la finca Rancho Grande y un valor mínimo de 2.30 para el tratamiento no fertilizado de la finca Chupillauta, dicho comportamiento se observa en la figura 4.

Al comparar las tres metodologías se observaron bajos coeficientes de determinación para el PCA 2000 en comparaciòn con el PIPEcv. Para el tratamiento fertilizado se obtuvo un coeficiente de determinación $R^{2}=0.011$ y para el tratamiento no fertilizado un coeficiente de determinación de $R^{2}$ $=0.039$ como se puede observar en la figura 5 . No se encontraron diferencias significativas entre las metodologías interactuando con los tratamientos ( $p=0.644$ a un nivel de significancia del $95 \%$ de probabilidad) pero si en interacción con las fincas $(p=0.019$ a un nivel de significancia del $95 \%$ de probabilidad; Tabla 2).

Al comparar las metodologías del PCA 2000 y el modelo matemático se encontró para las parcelas fertilizadas un coeficiente de determinación $R^{2}$ $=0.011 \mathrm{y}$ para las parcelas no fertilizadas un
$R^{2}=0.053$, tal como se observa en la figura 6 . El análisis de varianza mostró que en las dos metodologías, al interactuar con el factor finca, se encuentran diferencias significativas $(p=$ 0.024 a un nivel de significancia del $95 \%$ de probabilidad) y con el factor tratamiento no existen diferencias significativas ( $p=0.891$ a un nivel de significancia del 95\% de probabilidad; Tabla 3). Esto implica que la fertilización no generó cambios significativos en la producción de las plantaciones, pero, por su parte, el lugar donde se encuentra cada plantación sí influye en la cantidad de masa foliar que desarrollan los árboles.

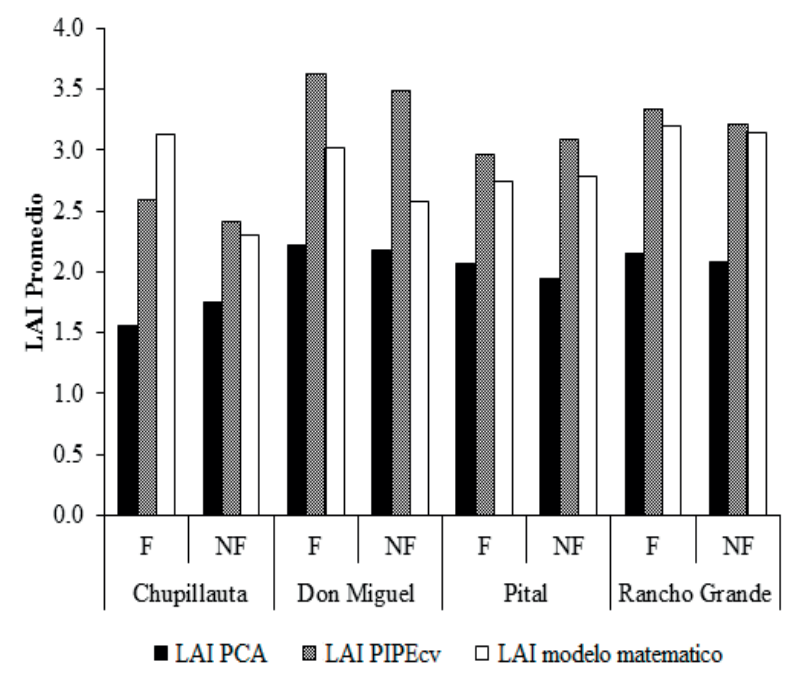

Figura 4. Comportamiento del índice de área foliar (IAF) en las parcelas fertilizadas (F) y no fertilizadas (NF) de Eucalyptus grandis W.Hill., en las fincas de la empresa Smurfit Kappa Cartón de Colombia 


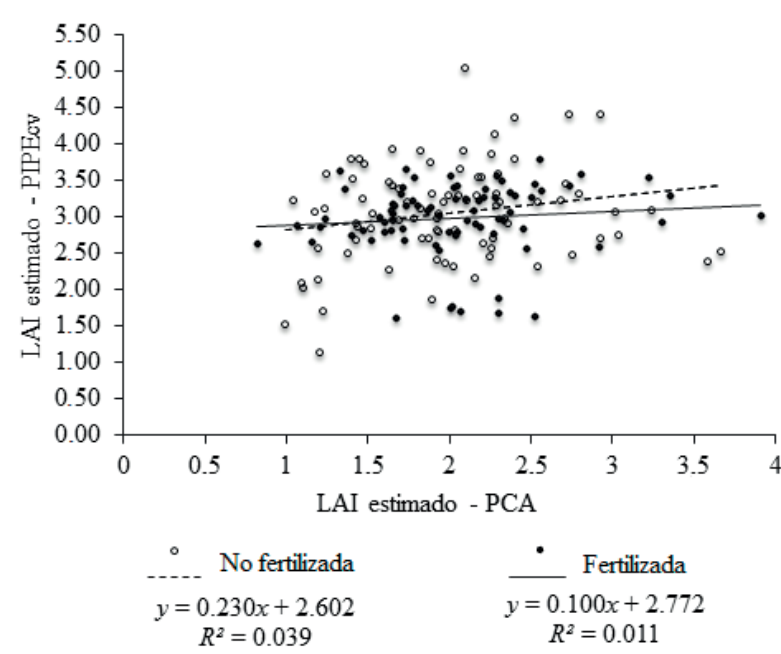

Figura 5. Relación entre el Plant Canopy Analyzer y el PIPEcv, para la estimación del índice de área foliar en Eucalyptus grandis W.Hill.

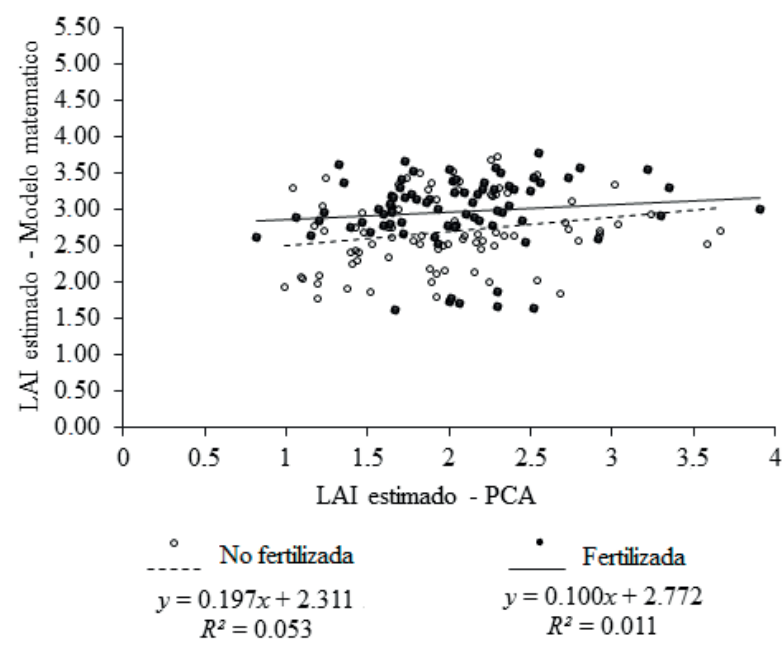

Figura 6. Relación entre el Plant Canopy Analyzer y el modelo matemático, para la estimación del índice de área foliar en Eucalyptus grandis W.Hill.

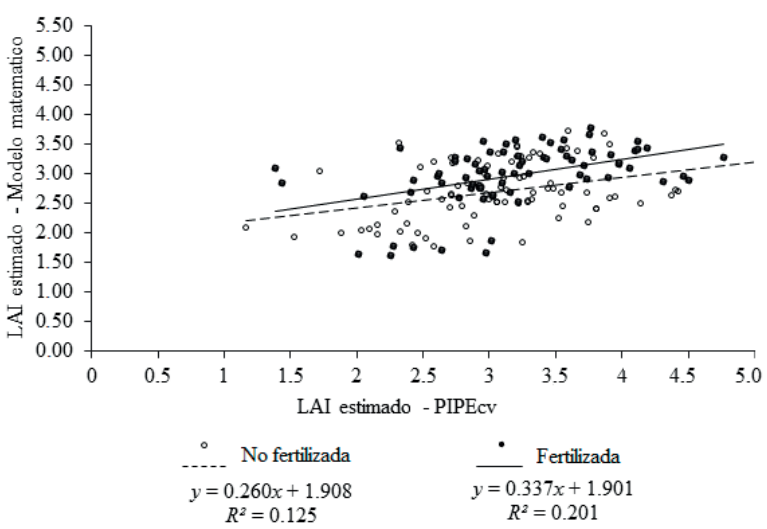

Figura 7. Relación entre el PIPEcv y el modelo matemático, para la estimación del índice de área foliar en Eucalyptus grandis W.Hill.
Tabla 2. Análisis de varianza Anova, relacionando el software PIPEcv y el Plant Canopy Analyzer para la estimación del índice de área foliar, en interacción con los tratamientos y las fincas, en las plantaciones de $E u$ calyptus grandis W.Hill. Diferencia entre el LAI estimado con el software PIPEcv y el Plant Canopy Analyzer

\begin{tabular}{|c|c|c|c|c|c|}
\hline Fuente & $\begin{array}{c}\text { SC tipo } \\
\text { II }\end{array}$ & gl & CM & $\mathbf{F}$ & p \\
\hline Modelo & 206.274 & 8 & 25.784 & 43.703 & 0.000 \\
\hline Finca & 6.048 & 3 & 2.016 & 3.417 & 0.019 \\
\hline Tratamiento & 0.126 & 1 & 0.126 & 0.214 & 0.644 \\
\hline $\begin{array}{l}\text { Finca * } \\
\text { tratamiento }\end{array}$ & 1.904 & 3 & 0.635 & 1.076 & 0.361 \\
\hline Error & 90.268 & 153 & 0.590 & & \\
\hline Total & 296.542 & 161 & & & \\
\hline
\end{tabular}

Tabla 3. Análisis de varianza que relaciona el modelo matemático con respecto a la metodología del Plant Canopy Analyzer, teniendo como factores las fincas y tratamientos, para la estimación del índice de área foliar en Eucalyptus grandis W.Hill. Diferencia entre el índice de área foliar estimada con el modelo matemático y el Plant Canopy Analyzer

\begin{tabular}{lrrrrc}
\hline \multicolumn{1}{c}{ Fuente } & \multicolumn{1}{c}{$\begin{array}{c}\text { SC tipo } \\
\text { II }\end{array}$} & \multicolumn{1}{c}{ gl } & \multicolumn{1}{c}{ CM } & \multicolumn{1}{c}{ F } & \multicolumn{1}{c}{$\boldsymbol{p}$} \\
\hline Modelo & 118.975 & 8 & 14.872 & 34.657 & 0.000 \\
Finca & 4.184 & 3 & 1.395 & 3.250 & 0.024 \\
Tratamiento & 0.008 & 1 & 0.008 & 0.019 & 0.891 \\
Finca * & 5.878 & 3 & 1.959 & 4.566 & 0.004 \\
tratamiento & & & & & \\
Error & 65.654 & 153 & 0.429 & & \\
Total & 184.628 & 161 & & & \\
\hline
\end{tabular}

Tabla 4. Análisis de varianza que relaciona el modelo matemático con respecto a la metodología del PIPEcv, teniendo como factores, los tratamientos fertilizados y no fertilizados en la plantación de Eucalyptus grandis W.Hill. Diferencia entre el LAI estimado con el modelo matemático y el PIPEcv

\begin{tabular}{|c|c|c|c|c|c|}
\hline Fuente & $\begin{array}{c}\text { SC tipo } \\
\text { II }\end{array}$ & gl & $\mathbf{C M}$ & $\mathbf{F}$ & $p$ \\
\hline Modelo & 33.021 & 8 & 4.128 & 9.127 & 0.000 \\
\hline Finca & 14.995 & 3 & 4.998 & 11.053 & 0.000 \\
\hline Tratamiento & 0.070 & 1 & 0.070 & 0.156 & 0.694 \\
\hline $\begin{array}{l}\text { Finca } * \\
\text { tratamiento }\end{array}$ & 4.639 & 3 & 1.546 & 3.420 & 0.019 \\
\hline Error & 69.192 & 153 & 0.452 & & \\
\hline Total & 102.213 & 161 & & & \\
\hline
\end{tabular}


Cuando se comparó la metodología del PIPEcv frente al modelo matemático se encontraron coeficientes más altos en relación con los mencionados con anterioridad: para el tratamiento fertilizado un coeficiente de determinación $R^{2}=0.0201$ y para el no fertilizado un coeficiente $R^{2}=0.125$, como se observa en la figura 7. Para estas metodologías, el análisis de varianza arrojó que, al igual que en el anterior, en interacción con las fincas hay diferencias significativas ( $p<<0.001$ a un nivel de significancia del $95 \%$ de probabilidad) y en la interacción con los tratamientos no hay diferencias significativas ( $p=0.694$ a un nivel de significancia del $95 \%$ de probabilidad; Tabla 4 ).

\section{MODELO MATEMÁTICO}

Se evaluó un modelo exponencial que describe el comportamiento de los datos de campo, el cual se usó para estimar el índice de área foliar de las diferentes parcelas. La figura 8 muestra la curva trazada en la masa de puntos por el modelo matemático, el eje $\mathrm{X}$ muestra los diámetros medidos en campo y emparejados con su respectiva área foliar en el eje Y.

El resultado de la tabulación y experimentación de diferentes modelos matemáticos dio como resultado un modelo exponencial, en el cual se tienen dos parámetros $a$ y $b$ que son constantes del modelo (2.91274630540194 y 0.100592754068322 , respectivamente; Ecuación 1).

$$
L A I=2.91274630540194 e^{0.100592754068322 D A P} \text { Ec. } 1
$$

donde, $L A I=$ índice de área foliar, $e=$ exponencial y $D A P=$ diámetro $(\mathrm{cm})$ a la altura de $1.30 \mathrm{~m}$.

El modelo encontrado tiene un error estándar de 13.22 un coeficiente de correlación $R=0.9066$ y un coeficiente de determinación $R^{2}=0.8220$; esto indica realizar estimaciones de índice de área foliar a través del modelo tiene una alta confiabilidad.

\section{DISCUSIÓN}

La información sobre el índice de área foliar en la especie E. grandis presentada en este estudio, aunque es preliminar en Colombia, se convierte en información importante con el fin de tomar decisiones acertadas sobre el manejo de la especie y en una línea base en la investigación de su comportamiento.

El análisis estadístico descriptivo permitió evidenciar que con el modelo matemático se obtienen valores menos dispersos con respecto a la media, en todos los sitios estudiados. Lo anterior se debe

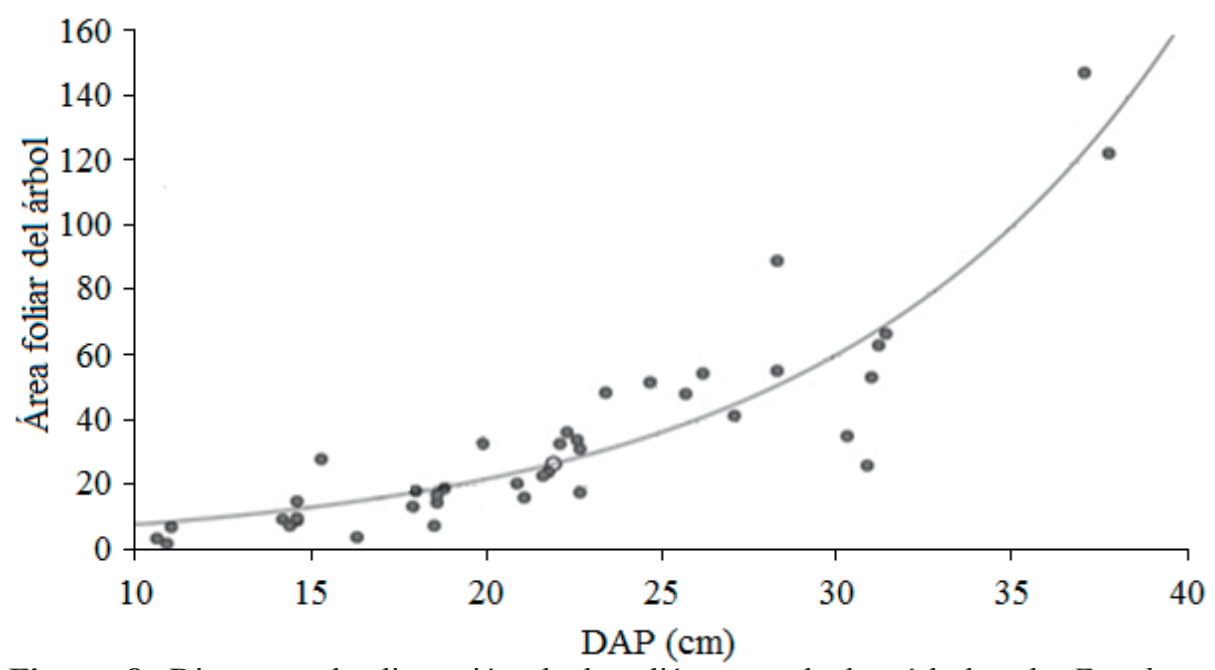

Figura 8. Diagrama de dispersión de los diámetros de los árboles de Eucalyptus sgrandis W.Hill. con respecto a su área foliar y curva trazada por el modelo matemático exponencial 
a que el modelo atribuye a cada diámetro de árbol un LAI estimativo o modelado, a diferencia de las otras dos metodologías que tienen en cuenta la cantidad de luz que las copas de los árboles dejan pasar para hacer la estimación del LAI. También se pudo establecer que con el PCA 2000 se obtienen datos más dispersos con respecto al promedio, lo cual puede deberse a las condiciones de luz ofrecidas por los sitios y a la alta sensibilidad del instrumento de medición a los cambios de luz o radiación solar.

Las posibles razones por las que no se encontraron diferencias significativas entre los tratamientos de fertilización en las fincas pudieron estar dadas por la edad que tenía la plantación al momento de las mediciones, ya que a los 7 años la plantación ya ha alcanzado su expansión foliar, lo que podría ser diferente si se evaluara al inicio de la siembra con tratamientos de fertilización y testigo.

El mayor índice de área foliar se presentó en la finca Don Miguel, para las metodologías del PCA 2000 y el PIPEcv. Para el modelo matemático, la finca con mayor LAI fue la finca Rancho Grande, debido a que este modelo, al estar en función de los diámetros de los árboles presentes en la parcela, sobrestima el LAI, y desafortunadamente en el inventario destructivo hubo poca representatividad de árboles con diámetros superiores a los $30 \mathrm{~cm}$, lo que pudo ocasionar errores de estimación. El menor

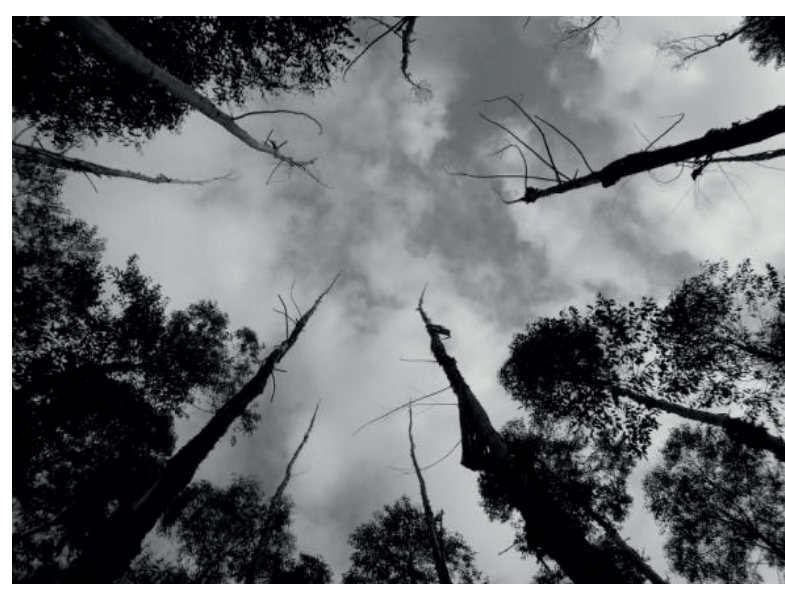

Figura 9. Árboles de Eucalyptus grandis W.Hill. afectados por la caída de rayos en la finca Chupillauta de la empresa Smurfit Kappa Cartón de Colombia índice de área foliar lo reportó la finca Chupillauta para las tres metodologías desarrolladas, como consecuencia de sucesos naturales como la caída de rayos que quemaron las copas de los árboles, lo que propició la apertura del dosel y, por ende, la subestimación del índice de área foliar (Figura 9).

Los valores obtenidos con las tres metodologías se encuentran dentro del rango reportado para las especies de hoja ancha a nivel mundial, que es entre 2 y 3 (Waring et al., 1992); aunque es de resaltar que los valores más altos corresponden especialmente a los estimados con el método de fotografía plana, debido a que este toma otras partes del árbol como si fueran hojas (Tabla 5). Para realizar una investigación rápida y de bajo costo, el método de fotografía plana es una metodología factible, ya que aunque los valores difieren de los encontrados con el PCA 2000, la fotografía plana ayuda a identificar las parcelas o tratamientos con mayor y menor índice de área foliar.

Tabla 5. Valores máximos, mínimos y promedio de índice de área foliar en Eucalyptus grandis W.Hill., estimados con las tres metodologías

\begin{tabular}{lrrr}
\hline \multicolumn{1}{c}{ Valor } & LAI PCA & LAI PIPEcv & $\begin{array}{r}\text { LAI modelo } \\
\text { matemático }\end{array}$ \\
\hline Máximo & 3.91 & 5.03 & 3.8 \\
Mínimo & 0.82 & 1.15 & 1.62 \\
Promedio & 2.01 & 3.12 & 2.83 \\
\hline
\end{tabular}

De acuerdo a lo expuesto por Peduzzi (2007), es posible observar que el índice de área foliar es un indicador de crecimiento, donde valores bajos indican baja productividad, caso que no se presenta en la plantaciones estudiadas, donde los promedios obtenidos de LAI se mueven en los rangos normales $\mathrm{y}$, en algunos casos, sobrepasan el valor de 3.0.

Los análisis estadísticos permitieron establecer que existe una correlación débil entre las tres metodologías; aunque se pudo observar un mayor grado de relación entre la metodología del PIPEcv y el modelo matemático, lo cual pudo deberse a que ambas metodologías tienen mayores errores al no ser un métodos de precisión como lo es la del PCA 2000. 
El modelo matemático encontrado tiene un buen ajuste, lo cual se evidencia en el coeficiente de determinación, el cual indica que el aumento del diámetro de los árboles se ve explicado en un $82.2 \%$ por el aumento del área foliar de los árboles estudiados. Lo anterior, es lógico si se tiene en cuenta que son las hojas las que realizan la fotosíntesis, proceso que se traduce en el crecimiento de los árboles. El modelo presentado puede ser una herramienta de uso fácil, rápido, confiable y económico para la estimación del índice de área foliar de individuos con diámetros desde los $10 \mathrm{~cm}$ hasta los $38 \mathrm{~cm}$, en el cual tienen baja representatividad los individuos con diámetros iguales o superiores a los $30 \mathrm{~cm}$, lo que hace que el modelo se limite a un rango de diámetros a fin de no sobrestimar o subestimar.

\section{CONCLUSIONES}

En general, el índice de área foliar en las cuatro fincas estudiadas estuvo en un promedio de 2.01 para el PCA 2000, 3.12 para el software PIPEcv y 2.83 para el modelo matemático, lo que responde a lo reportado para especies de hoja ancha a nivel mundial.

No se pudo hallar un factor de conversión que permitiera ajustar el método de estimación del índice de área foliar a través de fotografía plana con el estimado con el Plant Canopy Analyzer, debido a que estas dos metodologías son diferentes y los análisis estadísticos mostraron que no tenían una correlación fuerte.

Se encontró, a través de las tres metodologías, que existe relación entre el índice de área foliar estimado del modelo matemático frente al PIPEcv y del modelo matemático con respecto al PCA 2000 con una mejor relación entre las metodologías del PIPEcv y el modelo matemático.

Se pudo establecer que la finca con mayor índice de área foliar fue la finca Don Miguel, para los métodos del PCA 2000 y el software PIPEcv, y la finca con menor índice de área foliar fue la finca Chupillauta, para las tres metodologías empleadas.
El software PIPEcv es una herramienta útil y rápida de utilizar cuando se desea hacer una estimación en corto tiempo del LAI con una precisión media.

Aunque se han desarrollado algunas estimaciones de índice de área foliar en el mundo con fotografía hemisférica, no existe ninguna con fotografía plana que haya sido corroborada con métodos matemáticos y de campo especializados. Por esto, este trabajo se convierte en un aporte importante para la estimación de crecimiento con métodos rápidos y fáciles en campo, como lo son las fotografía digitales.

\section{AGRADECIMIENTOS}

Los autores agradecen a la Universidad del Cauca, la cual permitió la elaboración del convenio para el desarrollo de esta investigación, así como a la Facultad de Ciencias Agropecuarias donde se encuentra adscrita la docente encargada. Al Departamento de Investigación Forestal de la empresa Smurfit Kappa Cartón de Colombia, por brindar la oportunidad de realizar esta investigación en sus plantaciones forestales y por todo el apoyo logístico y humano para que la investigación fuese posible. A los ingenieros Marcela Zapata, Mauricio Zapata y John Byron Urrego, por su colaboración incondicional durante toda la investigación.

\section{REFERENCIAS BIBLIOGRÁFICAS}

Cabrelli, D., Rebottaro, S., \& Effron, D. (2006). Caracterización del dosel forestal y del microambiente lumínico en rodales con diferente manejo, utilizando fotografía hemisférica. Revista de Ciencias Forestales, 13, 17-25.

Canham, C.D., Denslow, J.S., Platt, W.J., Runkle, J.R., Spies, T.A., \& White, P.S. (1990). Light regimes beneath closed naopies and tree-fall gaps in temperate and tropical forest. Canadian Journal of Forest Research, 20, 620-631.

Chazdon, R.L., \& Fetcher, N. (1984). Photosynthetic light environments in a lowland 
tropical forest in Costa Rica. Journal of Ecology, 72, 553-564.

Hardy, J.P., Melloh, R., Koenig, G., Marks, D., Winstrall, A., Pomeroy, J.W., \& Link, T. (2004). Solar radiation transmission through conifer canopies. Agricultural and Forest Meteorology, 126, 257-270.

Hutchison, B.A., \& Matt, D.R. (1977). The distribution of solar radiation within a deciduous forest. Ecology Monographs, 47, 185-207.

Kellomaki, S., Oker-blom, P., \& Kuuluvainen, T. (1985). The effect of crown and canopy structure on light interception and distribution in a tree stand. En P.M.A. Tigerstedt, P. Puttonen \& V. Koski (eds.). Crop Physiology of Forest trees (pp. 107115). Helsinki: Helsinki University Press.

LI-COR. (1992). LAI-2000 plant canopy analyzer instruction/operatingmanual. Lincoln: LICOR, Inc. 217 p.
Lieberman, M., Lieberman, D., \& Peralta, R. (1989). Forest are nor just swischeese: Canopy stereogeometry of non-gaps in tropical forest. Ecology, 70, 550-552.

Peduzzi, A. (2007). Leaf Area Assessments of the overstory and understory vegetation in pine plantations located in south Georgia and North Florida, US. (M.Sc. Thesis). Raleigh: North Carolina State University. $42 \mathrm{p}$.

Poulson, P.L., \& Platt, W.J. (1989). Gap light regimes influence canopy tree diversity. Ecology, 70, 553-555.

Pukkala, T., Becker, P., Kuuluvainen, T., \& Oker-mlom, P. (1991). Predicting spatial distribution of direct radiation below forest canopies. Agricultural and Forest Meteorology, 55, 295-307.

Waring, R.H., Schroeder, P.E., \& R. Oren. (1992). Application of the pipe model theory to predict canopy leaf area. Canadian Journal of Forest Research, 12, 556-560. 